\title{
PAIN PATHWAYS IN ORTHOPAEDIC PRACTICE
}

\author{
J. D. G. Troup, M.R.C.S., L.R.C.P.* \\ Aberdeen
}

DesPite Steindler's (1959) admirable lectures on the subject, pain in orthopædic practice remains a difficult problem. To some extent the difficulty is exaggerated by the adoption of diagnoses based on pathological processes which are insusceptible of proof-diagnoses for which it is impossible to establish an association between symptoms and their alleged origin, let alone a direct causal link. For instance a nipped zygapophyseal synovial fringe may well cause acute symptoms, but as there is no means of examining the synovia of zygapophyseal joints, to make this the diagnosis is purely speculative. Nevertheless pathological guesswork of this sort is regrettably common, and when some of the latest advances in neurophysiology, for example the experimental evidence that higher brain activity may modify incoming sensory impulses, are added to the mixture, one even hears talk of such things as the psychosomatic causes of slipped disc. This is a pity because with adequate precision in both history taking and examination, particularly with regard to the mechanistic and neurological aspects, there is usually enough objective evidence at hand to obviate the need for guessing. Furthermore such evidence will probably fit in quite logically with what is known of the physiology of pain.

Unfortunately there is little reference made by the authorities to what in orthopædic medicine is a common clinical phenomenon. This is that pain pathways in general, and referred pain in particular, are pathological rather than physiological processes; or in other words local pathological changes have to be present before peripherally referred pain can be manifest. Healthy tissue may eventually become the seat of referred pain either because the primary lesion imposes a mechanical strain upon it, or because the primary lesion subjects it to unrelieved nervous irritation, or a combination of both. But until local changes of one sort or another have deprived it of health and caused an increased nervous discharge of impulses to originate from it, it cannot be the site for referred pain. A number of pathological factors,

* Present address: 5 Queen's Terrace, Aberdeen. of which time is one of the most significant, have to obtain before pain can be appreciated in tissues remote from a primary lesion, and when the pain is present, so too are palpably pathological changes. It is probably true that a peripheral pathway for pain can be excited centrally or by any stimulus proximal to it, but the pathway cannot be established in the first place without initial pathological changes in the periphery.

\section{The Appreciation of Pain}

The appreciation of pain is divided into two parts; first the sensory component-where it is and what it feels like, and secondly the affective component which dictates to what extent the pain occupies the subject's attention, the extent to which it interferes with normal living, and which has a strongly emotive quality. Few patients can distinguish the two components even if they are aware of them, but the physician must do so, and it is from the first that he will learn most. Unfortunately the first component can only be studied in isolation in patients who have had a prefrontal leucotomy. In these patients the nerve fibres which run from the higher areas of the brain to the message-carrying pathways in the spinal cord have been interrupted. Experiments are described (Melzack, 196r) in which these fibres have been demonstrated. We already know that higher brain function is capable of altering the intensity of nerve impulses produced by an injury, and there is now experimental proof that electrical stimulation of nerve fibres from higher brain areas can actually modify or suppress incoming messages from the cord. Melzack refers to the affective component of pain as the product of upbringing and environment, of previous experience of pain, and of present thoughts and emotions. Even heredity may have something to do with it. Some assessment of the second component has to be made, therefore, before the first, the sensory component, can be evaluated.

\section{The Transmission of Pain Sensation}

Pain can be transmitted by sensory nerve fibres of various sizes and with various conduction rates, 
but there is doubt as to whether there are nerve endings which exist only for pain reception. As recently as 1959, Bell, Davidson and Scarborough describe endorgans in skin which are distributed independently of the endorgans of touch and temperature, despite the evidence of Sinclair, Weddell and Zander (1952), Hagen, Knoche, Sinclair and Weddell (1953), and Gooddy (1957). Weddell (1960) found that in hairy (as distinct from glabrous) skin, free nerve-endings subserve pain as well as temperature, but that though free nerve-endings are not morphologically specific, they may be physiologically or biochemically specific. In 1959 Lele and Weddell were able to confirm the conclusions of Adrian (1926) that the "pain reaction must be determined by a summation of a number of impulses in the central nervous system'. But whether or not specific pain endorgans exist in the skin-and it is unlikely-there seems to be no evidence to suggest their presence in the deeper structures with which the orthopædist is concerned. The lesions from which pain originates are invariably the site of an increased discharge of nerve impulses, but whether or not these are interpreted as pain depends on a number of factors of which the spatio-temporal pattern of the impulses, and the patients' psychologically and conditionally induced receptivity for pain, are the most important.

Lewis and Pochin (1937) showed that painconducting nerve fibres sharply divided into fast and slow groups. After further experiments into the effects of pressure and asphyxia on nerves they showed (1938) that discriminatory sensations such as touch, deep pressure and recognition of position during passive movement are related to the fast fibres, whereas the sensation of tension from muscle contraction is related to the slowconduction-rate fibres subserving slow pain and temperature sense. Tissues such as skin and joints are better supplied with fast fibres for discriminatory sensation than are muscles and tendons which have a greater proportion of slow fibres. Pain in fast fibres generally ceases when the stimulus is removed, but in the slow fibres it may be prolonged after the stimulation stops. Bowsher (1957) has suggested that the direct spinothalamic system is responsible for fast pain, but the indirect spino-reticulo-thalamic system is responsible for the slow. Therefore, though the subjective interpretation of pain depends on both the spatio-temporal pattern of the impulses giving rise to it, and the patient's receptivity for it, it must also depend on the site of the pain and the conduction-rate of the fibres conveying the impulses. At the risk of over-simplification it can be said that joints give rise to fast pain which is unlikely to persist beyond the withdrawal of stimulation, whereas muscles and tendons afje more likely to exhibit slow pain which can persist. Therefore the site of the pain, the quality of the pain and its temporal pattern as distinct from ids quantitative and effective aspects, may yietd useful information as to its pathologic 1 origin.

\section{The Evaluation of Pain}

The first problem in evaluating pain is to assess its affective component, the patient's receptivify for it. The second is to decide whether the paif coincides with the apparent cause. The pain on spraining an ankle is immediate and obvious and once the initial shock has worn off, the pais is not continuous so much as a response to prevent movement; in fact it coincides with the stimulus. A sustained contraction of a muscle results in pat which does not disappear completely on relaxatic but continues for a short period, while musculat activity for which an individual is not in training $\vec{g}$ though at the time painless, may cause a lowered pain threshold in the muscle on the following day. These instances are of what I might call normal pain in a physiological sense, though their origias are in pathological tissue changes. In distinctiog to these physiologically normal pain reactions are those in which the pain begins without any ofien cause. For instance, skin and subcutanêus tissue which becomes hyperalgesic, pain wis begins in a muscle at rest after normal activit and cases of referred pain, are examples of pain of

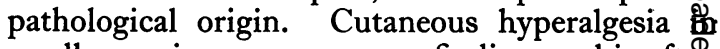
small areas is a very common finding, and is ofte a phenomenon which behaves much like referreg pain (Feinstein, Langdon, Jameson and Schilles, 1954). Lewis and Kellgren (1939) demonstrated it in patients with angina. In practice it is found within the dermatome of the nerve segment supplying the source of irritation. Very often the area is palpable, there being some induration with increased sweat-gland activity. It is probably neuro-circulatory failure but one can only speculąe as to the mechanism. Being a derangement of nerve activity it is accompanied by hypoæsthes not hyperæsthesia. Similar lesions are often found in subcutaneous or muscle tissue and have been variously described according to their nature and extent, for instance panniculitis (Baker, 195 r) fibrositis or myogelosis (Steindler, I959), and adiposus dolorosa. They have one thing in conas mon with the cutaneous lesion, they are $\mathbb{P}$ hyperalgesic and hypoæsthetic, and they have ore incidental thing in common with each other, theg occur in tissues with high fat content, which explains why they are found in such muscles the gluteals and trapezius, and in subcutaneouis tissue. 


\section{The Origin of Deep-Structure Pain}

Pain in deep structures such as muscle can be due to local causes (which are probably the commonest), secondly to lesions affecting the conductivity of the nerve fibres supplying them, or thirdly it can arise centrally.

The commonest local cause of pain in a muscle is sustained tension; just as a sustained maximal contraction of a muscle produces pain, so sooner or later does an unrelieved minimal contraction. Hypertension can arise in a muscle from a number of causes such for example as fatigue, static and kinetic postural faults, weakness of the opposing muscle or muscle group, inflammation in a joint on which the muscle acts, or a mechanical imbalance due to a deformity.

Of the lesions affecting the conductivity of a nerve and causing referred pain, those arising from a source of irritation such as an inflamed joint or fibrositic nodule are the commonest. Referred pain almost always arises from a source in the same nerve segment to which it is referred (Feinstein and others, 1954; Kellgren, 1938, 1939; Lewis and Kellgren, 1939), and is indistinguishable in character whether the source of irritation is in somatic or visceral tissue (Kellgren, 1940). When the cause of muscle pain is local there will be clinical signs to account for it, but if the pain is referred it is usually possible to locate the trigger-point which is the source of reflex irritation, as well as the local clinical signs. The significant feature of referred pain is not that it is limited most often to the same nerve segment as its origin, but that it is limited to a single part of that segment. Severe pain of short duration which shoots down a limb is more likely to be due to the consquence of inflammation in mechanically related tissues, examples being pain at the wrist from a radiohumeral lesion, or pain at the deltoid insertion from an acromioclavicular lesion, than to a purely neuropathological cause. Persistent referred pain however is almost always limited topographically to a part of the neurosegment, hardly ever involving the whole area supplied in the segment. This, combined with the comparative ease by which the site of referred pain can be localized by palpation, leads to the hypothetical conclusion that changes must be present before referred pain can be manifest.

Lesions of nerve fibres themselves can also cause pain. But such pain cannot be predictable when the essence of the lesion is damage to the nerve fibre. For instance pressure on a healthy nerve causing the foot or the hand to 'go to sleep" is the familiar experience of paralysis due to complete loss of conduction. If it is maintained for long enough, there will ultimately be a necrosis of the nerve fibres. But a less intense pressure or any other noxious influence on a nerve fibre such as exposure to cooling or anoxæmia, causes an intensified electrical discharge from the nerve. Nathan (1953) showed that small painless lesions became painful when the nerve concerned was compressed. Kellgren, McGowan and Hughes (I949) found that when pain in a limb was induced by cooling it, the tissues at the source of pain were hyperalgesic, and that the same process on patients with painful hyperalgesic extremities produced a noticeably lowered pain threshold. The same effect is found in tissues which are the site of referred pain (Lewis and Kellgren, 1939; Hardy, Wolff and Goodell, I94I; Feinstein and others, I954), and in tissues which are subjected to vasoconstriction (a reflex phenomenon frequently encountered in association with the peripheral neuropathies). But whatever noxious factor is responsible for interfering with conductivity it is apparent that though it causes an intensified discharge of impulses, the affected tissues becoming hyperalgesic, there is not an increased sensitivity to all sensation. Bigelow, Harrison, Goodell and Wolff (1945) found that the hyperalgesia associated with peripheral neuropathy was explained by a depression of the threshold for slow pain and an elevation of that for fast pain.

It would seem therefore that damage, resulting in an intensified discharge of impulses, to fastconducting fibres may cause paræsthesiæ and hypoæsthesia, but it does not cause pain; whereas in slow-conducting fibres the response is most likely to be interpreted as painful, though the quality of the pain may be described as burning, cold, gripping and so on, according to the pattern of intensity. When there is an established source of irritation within a nerve segment, it causes hyperalgesia and hypoæsthesia within the segment, but whether paræsthesiæ in tissues supplied by fast-conducting fibres, or pain in those supplied by slow fibres, are present, depends upon whether local pathological changes or external noxious factors prevail.

Persistent pain, hyperalgesia, paræsthesiæ and hypoæsthesia, and muscle weakness in a limb, are often encountered in association with spinal lesions. The symptoms are consistent with minor degenerative changes involving nerve trunks, changes which, judging by their response to treatment, are apparently reversible. Probably what happens in such cases is that a state of chronic tissue inflammation involving the region of the intervertebral foramen, secondary to an intervertebral lesion, surrounds the nerve trunk and its nutrient artery in conditions of circulatory stasis. This probably arises from local conditions of altered $p \mathrm{H}$ and tissue stagnation, due to axon reflex vasoconstriction and obstruction to drainage 
secondary to inflammatory œdema and lack of mobility. This would account for relief of symptoms on restoration of normal mechanical function (Troup, 196r). These same conditions probably obtain also in cases of root-sleeve fibrosis (Frykholm, 195I). Very often these patients are diagnosed as having pressure on a nerve trunk from a protruded disc, but this would be likely only if it were possible to prove beyond reasonable doubt that the pressure was actually caused by the protruded disc and that the pressure actually caused the symptoms (neither of which is generally possible), and if it were true that pressure on a nerve invariably caused pain (and it is not). (Kelly, 1956, 1960.)

Pain in deep structures arising centrally can be a very difficult problem because it is more or less independent of peripheral pathways. If the pathway can be interrupted surgically, the relief is only temporary until the pain pattern is reestablished in a new channel. It has even been known to re-establish itself after cortical topectomy and leucotomy (Gooddy, 1957). This phenomenon occurs in cases with 'phantom pain' in an amputated limb. However the significance of this is that there has been a lesion peripherally before the amputation was necessary, the section of the nerves themselves quite possibly contributing to the phenomenon. It may be true to say that the pain is initiated centrally, but when considering the ætiology of the pain, the peripheral factors cannot be wholly discounted.

By way of illustration, the following case is of interest. Mr. R., aged 4I, a working farmer, slipped while mucking out his byre, falling heavily onto his right buttock. He was straightaway disabled by pain, and when seen within a few hours, complained of pain in his low back and right calf, violent pain deep in his low back on coughing, pain at the back of the right hip on right leg weight-bearing, and finally numbness on the lateral side of calf and foot. He had first hurt his back six years previously and developed right leg pain during a prolonged recovery, and since then had been subject to some occasional aching in low back and leg. On examination he was crippled with pain, being unable to take any weight on the right leg. Examination was difficult owing to pain, but it was obvious that there was an acute lesion involving both right lumbosacral intervertebral and sacroiliac joints and the lumbosacral disc. Local anæsthetic into the sacroiliac joint instantly relieved the pain in the calf and the pain of right leg weight-bearing. Two days later he was symptom-free and the numbness had almost gone. The pain on coughing had been due to an inflamed disc, the back pain was partly referred from the intervertebral lesion and partly from the sacroiliac, and the calf pain was obvious referred from the sacroiliac joint; while numbness was almost certainly due to compressie of the nerve trunk by inflammatory œdema.

This was an acute injury with a relatively straightforward previous history. Apart from the differentiation of the various pains which was sharply clarified, he illustrated the phenomenon of a pathway for referred pain being establish centrally by the previous incidents with ther residual pathological changes. Even though the original leg pain may not necessarily have been $5^{a}$ referred one (it may have been due to prolonged disability having caused mechanical strain on thae leg and therefore circulatory stasis) the pathwigy for the referred pain was quickly occupied afer this particular injury. We do not, after att, normally expect to have such a crippling coros plexity of pain so soon after falling - no matterr how heavily. Generally speaking referred paith behaves in the way that experimental evidence $\vec{\sigma}$ ff it has shown, but there is usually evidence off some previous incident, however slight, involving the tissues in which it occurs. The same is true of peripheral neuropathies whatever their pathelogical origin. Pain which travels peripherally over a prolonged period such as the familiar syndrome of pain in neck, shoulder, arm, forearm oged eventually wrist, follows a route dictated by mechanical stresses and strains, but eventualtyoa nervous pathway can develop centrally, whi links them; and in an advanced case one fin trigger points arising anywhere between segmeros $\mathrm{C}_{5-6}$ and $\mathrm{D}_{3-4}$, irritation of any of which sends the pain and paræsthesiæ shooting down t $\overrightarrow{8 \mathrm{Be}}$ arm.

\section{Conclusion}

We have seen that the qualitative interpretation of pain depends on the space-time pattern of the impulses, the site of the pain and the conductiograte of the fibres conveying the impulses, as we्dl as the cause of the increased discharge of impulsegs interpreted as pain. Though Melzack has beęn able to modify or suppress incoming sensomy impulses by stimulation of the higher centres experimentally, in practice it is the rule for activity in the higher centres only to exaggerate or depress the interpretation of impulses, not to modify either the apparent site of origin or the quality of the sensations arising from it. It seents unlikely that higher brain activity can alter the spatio-temporal pattern of impulses, or the site $\frac{\partial f}{f}$ the nerve endings from which the impulses aris. Therefore, if this much is true, the quality of pain and its source are of first-hand diagnostgc importance. If the patient complains of pain, its origin can usually be discovered. But casưfl 
examination of a given lesion reveals nothing of whether the individual has symptoms from it, even if it is hyperalgesic. There is one thing about pain which is inescapable, it can never be discounted. To dismiss pain as psychosomatic or functional is to ignore the mechanism by which pain arises. Pain cannot be initiated centrally because there is always some clinico-pathological peg on which pain must first be hung peripherally, before the activity of the higher centres can influence the interpretation of sensory impulses from it, and establish a pathway for the pain. If the lesion cannot be found it is the physician rather than the patient who is to blame.

\section{REFERENCES}

Adrian, E. D. (1926): The Impulses Produced by Sensory Nerve Endings, F. Physiol (Lond.), 62, 33.

BAKER, D. (I95 I): Panniculitis, Lancet, ii, 753.

Bell, G. M., Davidson, J. N., and Scarborough, H. (1959): Textbook of Physiology and Biochemistry, 4th edn. Edinburgh: E. \& S. Livingstone.

Bigelow, N., Harrison, L., Goodell, H., and Wolff, H. G. (i945): Studies on Pain: Quantitative Measurements of Two Pain Sensations in the Skin, with reference to the Nature of the Hyperalgesia of Peripheral Neuritis, 7 . Clin. Invest, 24, 503.

Bowsher, D. (1957): Termination of the Central Pain Pathway in Man: the Conscious Appreciation of Pain, Brain, 80, 606 .

Feinstein, C., Langdon, J. N. K., Jameson, R. M., and Schiller, F. (1954): Experiments on Pain referred from Deep Somatic Structures, $\mathcal{F}$. Bone $\mathscr{f}$ t. Surg., 36, $98 \mathrm{I}$.

Fryкholm, R. (I95I): Cervical Nerve Root Compression resulting from Disc Degeneration and Root Sleeve Fibrosis, Acta. Chir. Scand., Supplement 160.

GoodDy, W. (1957): On the Nature of Pain, Brain, 80, i 18.

Hagen, E., KNoche, H., Sinclair, D. C., and Weddell, G. (I953): The role of Specialised Nerve Terminals in Cutaneous Nerve Sensibility, Proc. roy. Soc. Med., I4I, 279.

Hardy, J. D., WolfF, H. G., and Goodel.l, H. (I94I): Studies on Pain: Observations on the Hyperalgesia associated with Referred Pain, Amer. F. Physiol., 133, 3 I6.

Kellgren, J. G. (1938): Observations on Referred Pain arising from Muscle, Clin. Sci., 3, I75.

- (r939): On the Distribution of Pain arising from Deep Somatic Structures with Charts of Segmental Pain areas, Clin. Sci., 4, 35 .

(1940): Somatic Simulating Visceral Pain, Clin. Sci., 4, $3 \circ 3$.

_- McGowan, A. J., and Hughes, E. S. R. (1949): On Deep Hyperalgesia and Cold Pain, Clin. Sci., 7, I3.

Kelly, M. (1956): Is Pain due to Pressure on Nerves? Spinal Tumours and the Intervertebral Disc. Neurology (Minneap.), 5, 178.

(1960): Does Pressure on Nerves Cause Pain?, Med. F. Aust., I 18.

Lele, P. P. G., and Weddell, G. (1959): Sensory Nerves of the Cornea and Cutaneous Sensibility, Experimental Neurology, $\mathbf{1}, 334$.

Lewis, T., and Kellgren, J. G. (r939): Observations relating to Referred Pain, Visceromotor Reflexes and Other Associated Phenomena, Clin. Sci., 4, 47.

- and Pochin, E. E. (1937): The Double Pain Response of the Human Skin to a Single Stimulus, Clin. Sci., 3, 67 .

- (1938): The Effect of Asphyxia and Pressure on Sensory Nerves of Man, Clin. Sci., 3, I41.

Melzack, R. (196r): Perception of Pain, Scientific American, 204, $4 \mathrm{I}$.

Nathan, P. W. (1953): Nervous Discharges from Small Painless Lesions in Skin and Muscle, f. Neurol. Neurosurg. Psychiat., 16, 19.

Sinclair, D. C., Weddeli, G., and Zander, E. (I952): The Relationship of cutaneous sensibility to Neurohistology in the Human Pinna, F. Anat. (Lond.), 86, 402.

STEINDleR, A. (1959): Lectures on the Interpretation of Pain in Orthopedic Practice. Springfield, Illinois: Charles C Thomas.

Troup, J. D. G. (I961): The Significance of Disc Lesions, Lancet, ii, +3 .

Wedpell, G. (1960): Studies related to the Mechanism of Common Sensibility. Ch. VII in Cutaneous Innervation. Ed. Montagna. W., Oxford, London, New York, Paris: Pergamon. 